\title{
Test-retest reliability of and age-related changes in the subjective postural vertical on the diagonal plane in healthy subjects
}

\author{
Kazuhiro Fukata ${ }^{1,2} \cdot$ Kazu Amimoto $^{2} \cdot$ Daisuke Sekine $^{1} \cdot$ Yuka Ikarashi $^{1} \cdot$ Yuji Fujino $^{1} \cdot$ Masahide Inoue $^{1}$. \\ Mamiko Inoue $^{1} \cdot$ Shunichi Ishihara $^{3} \cdot$ Hidetoshi Takahashi $^{4}$. Shigeru Makita ${ }^{4}$
}

Published online: 16 November 2018

(C) The Psychonomic Society, Inc. 2018

\begin{abstract}
The subjective postural vertical (SPV) reflects gravity perception when the eyes are closed. Changes in the SPV on both the frontal and sagittal planes occur in response to neurological disorders and aging; however, these changes on the diagonal plane are unclear. Here we examined test-retest reliability $(n=16)$ of and age-related changes $(n=38)$ in the SPV on the diagonal plane. Subjects sat on an electrical vertical board (EVB), which was used to measure the SPV on the diagonal plane. An experimenter controlled and moved the EVB seat at a constant speed on the diagonal plane and measured the seat's tilt using a digital inclinometer when subjects verbally reported that they had reached a true vertical position. Measurement was performed for eight trials, and the mean (tilt direction) and standard deviation (variability) were calculated. To determine test-retest reliability, the same experimenter repeatedly measured the SPV 1 week later. To assess age-related changes, tilt direction and variability were compared between the young $(n=20)$ and elderly $(n=18)$ groups. Test-retest reliability on the right and left diagonal planes was 0.61 or more. Moreover, tilt direction on the right diagonal plane - but not on the left diagonal plane - indicated a significant diagonally backward deviation in the elderly group compared with that in the young group. Variability was significantly higher in the elderly group on both planes. SPV measurement on the diagonal plane was indicated, and age-related changes were identified. Thus, future studies should assess the potential clinical applications of SPV in neurological disorders.
\end{abstract}

Keywords Perception and action $\cdot$ Neuropsychology $\cdot$ Cognitive neuroscience

\section{Introduction}

The subjective postural vertical (SPV) indicates cognitive awareness of postural orientation and is assessed using motordriven machines (Bisdorff, Wolsley, Anastasopoulos, Bronstein, \& Gresty, 1996; Karnath, Ferber, \& Dichgans,

Kazuhiro Fukata

fkazuhiro.68@gmail.com

1 Department of Rehabilitation Center, Saitama Medical University International Medical Center, 1397-1 Yamane, Hidaka, Saitama 350-1298, Japan

2 Department of Physical Therapy, Tokyo Metropolitan University, 7-2-10 Higashi-Ogu, Arakawa-ku, Tokyo 116-8551, Japan

3 Department of Human Sciences, Faculty of Human Sciences, Bunkyo University, 3337 Minamiogishima, Koshigaya, Saitama 343-8511, Japan

4 Department of Rehabilitation, Saitama Medical University International Medical Center, 1397-1 Yamane, Hidaka, Saitama 350-1298, Japan
2000) or a non-motorized paradigm (Barbieri, Gissot, \& Pérennou, 2010; Baggio et al., 2016; Pérennou et al., 2008) that can tilt on the frontal or sagittal plane. In such tests, subjects report experiencing true vertical positioning when their eyes are closed. Hence, the SPV reflects gravity perception of the body.

Interestingly, an abnormal tilt and variability on the frontal plane are experienced in patients with pusher behavior or a vestibular deficit. Patients with cerebrovascular disease in general do not manifest an impaired SPV; however, those with pusher behavior, a severe postural control disorder in some patients with cerebrovascular disease, show a tilted SPV on the frontal plane while sitting (Karnath et al., 2000; Pérennou et al., 2008) and standing (Bergmann et al., 2015). In the study of Bergmann et al. (2016), the variability in the SPV on the frontal and sagittal planes showed a lower sensitivity in patients with pusher behavior than in those without pusher behavior and in healthy subjects. Bisdorff et al. (1996) reported no abnormal tilt of SPV in patients with vestibular deficits, but they did find reduced sensitivity.

Recently, the SPV on the sagittal plane has been shown to deviate backward with age (Barbieri et al., 2010). In addition, 


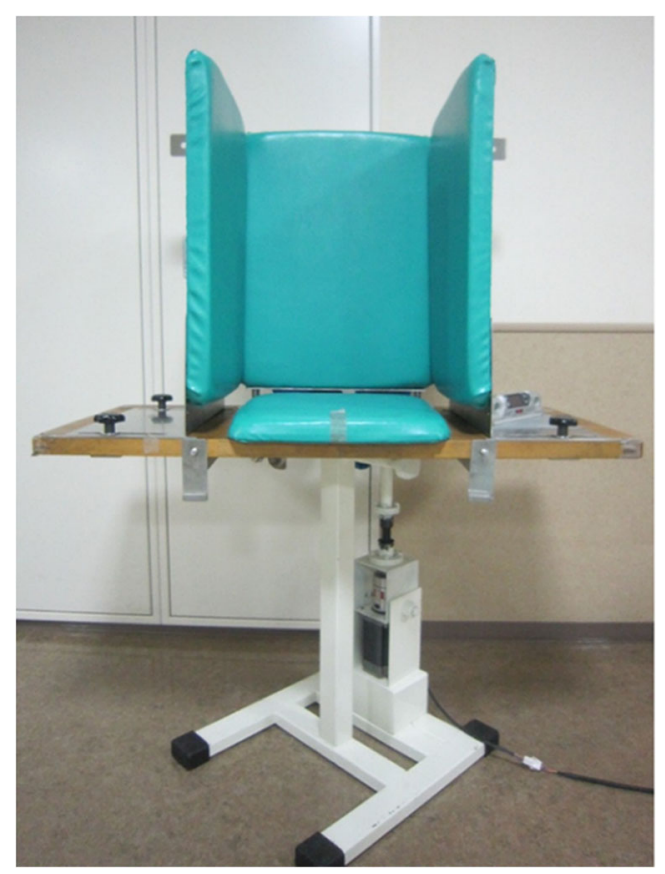

Fig. 1 Electrical vertical board

the variability in the SPV on the frontal plane has been reported to be significantly higher in elderly subjects than in younger subjects (Fukata et al., 2017). These results indicate that the precision or sensitivity of SPV declines with age; hence, it is important to determine the effect of aging on vertical perception.

Although previous studies on SPV were mainly concerned with the frontal and sagittal planes, activities of daily living require movement on the combined plane as well, such as that when moving from bed to a wheelchair. In patients with pusher behavior, resistance to combined plane movement, such as gliding the buttocks on the mattress toward the nonparetic side and transferring from the bed onto the wheelchair toward the nonparetic side, has also been observed (Karnath and Broetz, 2003). Therefore, it is necessary to assess the SPV not only on the frontal plane but also on the combined plane in patients with pusher behavior. In other words, the SPV can change in response to cerebrovascular disease on the diagonal plane in a similar manner to changes in the frontal and sagittal planes.

We developed an electrical vertical board (EVB) to measure the SPV on the diagonal plane. As this machine enables constant adjustments to the rotational speed, its measurement accuracy is thought to be high. However, there can be a time lag between the subject and experimenter in the SPV measurement; hence, the experimenter pushes the button to stop the machine when the subject believes that he or she is in an upright position. In addition, the obtained value itself may fluctuate; therefore, the SPV is a subjective sensation, and it is important to investigate the reliability of SPV measurement on the diagonal plane. a

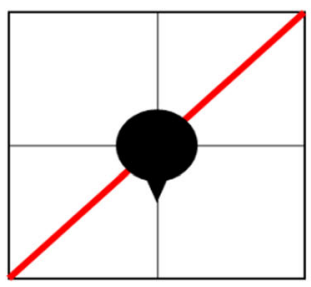

d

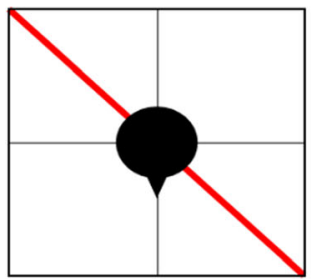

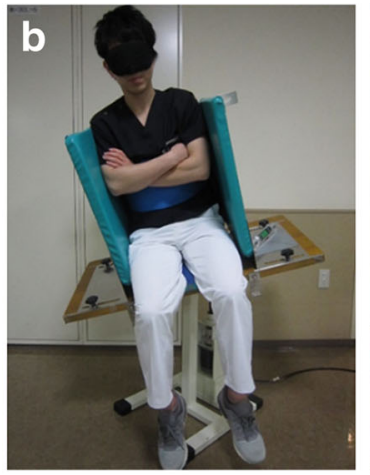
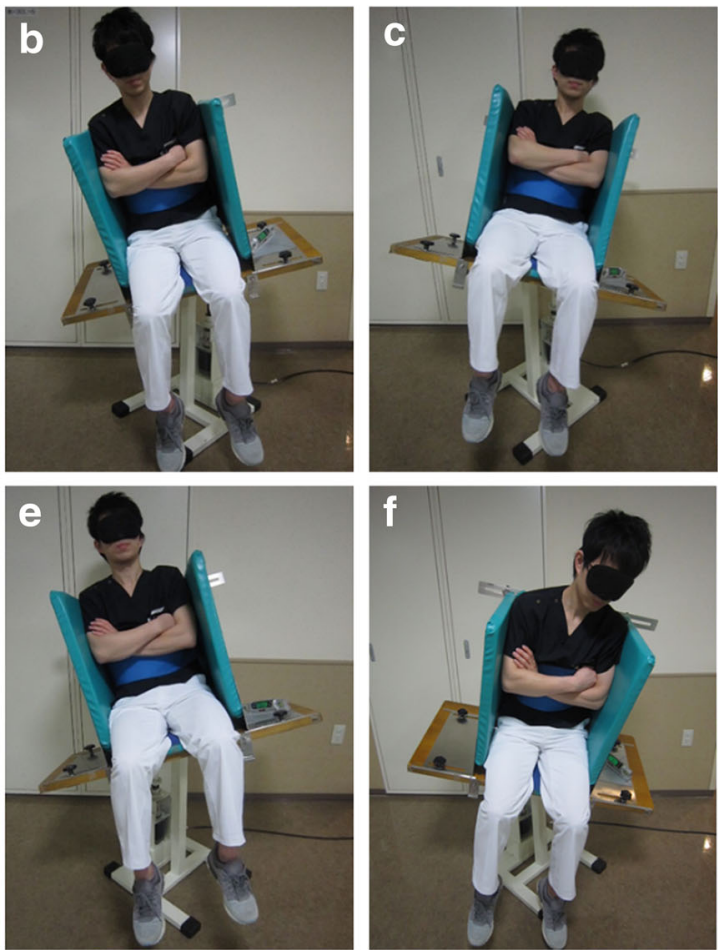

Fig. 2 Measurement conditions for subjective postural vertical on the diagonal plane. (A) Condition on the right diagonal plane; (B) starting position for the diagonally forward tilt toward the right side; (C) starting position for the diagonally backward tilt toward the left side; (D) condition on the left diagonal plane; (E) starting position for the diagonally forward tilt toward the left side; and (F) starting position for the diagonally backward tilt toward the right side 
Table 1 Demographics of 16 healthy subjects

\begin{tabular}{ll}
\hline Variable & Total $(\mathrm{n}=16)$ \\
\hline Age (years) & $37.1 \pm 13.7$ \\
Age (range, years) & $22-67$ \\
Gender (male/female) & $8 / 8$ \\
Height $(\mathrm{cm})$ & $165.2 \pm 7.8$ \\
Body weight $(\mathrm{kg})$ & $58.6 \pm 11.1$ \\
BMI $\left(\mathrm{kg} / \mathrm{m}^{2}\right)$ & $21.3 \pm 2.6$ \\
\hline
\end{tabular}

*Values are presented as mean \pm standard deviation.

$B M I$ body mass index

The present study aimed to clarify the test-retest reliability of and age-related changes in the SPV on the diagonal plane.

\section{Methods}

\section{Subjects}

Subjects with no history of bone and joint diseases, neurological disorders, psychiatric disorders, or dementia were included in this study. The SPV on the diagonal plane was assessed in 38 healthy subjects who were divided into two groups for the analysis of age-related changes, following the protocol of Barbieri et al. (2010): 20 young subjects (aged $<50$ years) and 18 elderly subjects (aged $\geq 50$ years). In addition, a second SPV measurement to determine test-retest reliability was performed in 16 of 38 healthy subjects who provided informed consent. This study was approved by the institutional review board of the Saitama Medical University International Medical Center (approval number: 16-189). All subjects provided written informed consent before their participation. Further, all subjects walked without a cane, had no history of falls, and were right-handed. We collected the following demographic data: age, gender, height, body weight, and body mass index (BMI).

\section{SPV measurement and procedure}

The SPV was measured using an EVB (Pair Support Corporation, Saitama, Japan) (Fig. 1), which is a motordriven machine that can be set to an inclination speed. In addition, the lateral wall width can be adjusted in accordance with the subject's body. Subjects sat on the EVB without contact with the ground; their trunks were fixed, their arms were crossed in front of their chest, and the positions of their head and legs were not fixed. The SPV was measured using the experimenter adjustment method in which the experimenter operated the EVB. The experimenter controlled the EVB seat by tilting it toward a vertical position from a position of $15^{\circ}$ or $20^{\circ}$, moving it at a rate of $1.5^{\circ} / \mathrm{s}$. The tilt of the seat when the subjects verbally reported that they had reached a true vertical position was measured using a digital inclinometer. Eight trials were performed in an $\mathrm{ABB} A \mathrm{BAAB}$ sequence so that the starting position and angle would be pseudorandom. In trials for the SPV, the subjects wore opaque goggles to cut off visual information. A true vertical position was considered to be $0^{\circ}$, whereas diagonally forward and diagonally backward tilts were regarded as positive and negative, respectively. SPV measurement was performed starting from either the left back or the right front (right diagonal plane) and from either the right back or the left front (left diagonal plane) (Fig. 2). Measurement conditions were randomly selected. The mean (tilt direction) and standard deviation (variability) in the eight trials were calculated. Tilt direction was defined as the direction of inclination on the basis of the body's vertical perception. On the right diagonal plane, the negative value referred to the diagonal backward tilt toward the left side, whereas the positive value pertained to the diagonal forward tilt toward the right side. On the left diagonal plane, the negative value referred to the diagonal backward tilt toward the right side, whereas the positive value pertained to the diagonal forward tilt toward the left side.

To clarify test-retest reliability, the SPV on each plane was measured in duplicate by the same experimenter at a 1-week interval between the first and second assessments. No feedback on performance was provided to subjects until completion of the two SPV measurements.

Table 2 Test-retest reliability of tilt direction and variability on the right and left diagonal planes

\begin{tabular}{|c|c|c|c|c|c|c|c|}
\hline Variable & Condition & First assessment $\left({ }^{\circ}\right)$ & Second assessment $\left({ }^{\circ}\right)$ & ICC $(95 \% \mathrm{CI})$ & $\operatorname{SDd}\left({ }^{\circ}\right)$ & $\operatorname{SEM}\left({ }^{\circ}\right)$ & $\operatorname{MDC}_{95}\left({ }^{\circ}\right)$ \\
\hline \multirow[t]{2}{*}{ Tilt direction } & Right diagonal & $-0.3 \pm 1.2$ & $-0.7 \pm 1.2$ & $0.69(0.33-0.88)$ & 0.9 & 0.7 & 1.8 \\
\hline & Left diagonal & $-0.1 \pm 1.2$ & $-0.4 \pm 1.0$ & $0.71(0.36-0.88)$ & 0.8 & 0.5 & 1.5 \\
\hline \multirow[t]{2}{*}{ Variability } & Right diagonal & $1.9 \pm 0.6$ & $2.1 \pm 0.5$ & $0.61(0.19-0.84)$ & 0.5 & 0.3 & 0.9 \\
\hline & Left diagonal & $2.2 \pm 1.0$ & $2.1 \pm 0.8$ & $0.63(0.22-0.85)$ & 0.8 & 0.6 & 1.6 \\
\hline
\end{tabular}

ICC intraclass correlation coefficient, 95\% CI 95\% confidence interval, SDd standard deviation of the difference between two measurement scores, SEM standard error of measurement, $M D C_{95}$ minimal detectable change with $95 \% \mathrm{CI}$ 
Table 3 Test-retest reliability of all trials on the right and left diagonal planes

\begin{tabular}{|c|c|c|c|c|c|c|c|}
\hline Variable & Trial & First assessment $\left({ }^{\circ}\right)$ & Second assessment $\left({ }^{\circ}\right)$ & ICC (95\% CI) & $\operatorname{SDd}\left({ }^{\circ}\right)$ & $\operatorname{SEM}\left({ }^{\circ}\right)$ & $\operatorname{MDC}_{95}\left({ }^{\circ}\right)$ \\
\hline \multirow[t]{8}{*}{ Right diagonal } & $1 \mathrm{st}$ & $-1.3 \pm 2.6$ & $-1.1 \pm 2.5$ & $0.74(0.40-0.90)$ & 1.8 & 1.3 & 3.6 \\
\hline & 2nd & $0.1 \pm 2.3$ & $0.0 \pm 2.2$ & $0.78(0.48-0.92)$ & 1.5 & 1.1 & 3.0 \\
\hline & $3 \mathrm{rd}$ & $0.2 \pm 2.3$ & $0.4 \pm 2.5$ & $0.56(0.09-0.82)$ & 2.3 & 1.6 & 4.5 \\
\hline & 4 th & $-0.5 \pm 1.9$ & $-1.2 \pm 1.6$ & $0.46(0.02-0.77)$ & 1.8 & 1.3 & 3.5 \\
\hline & 5 th & $0.2 \pm 2.1$ & $-0.3 \pm 3.2$ & $0.53(0.07-0.81)$ & 2.6 & 1.8 & 5.1 \\
\hline & 6th & $-0.6 \pm 2.1$ & $-1.0 \pm 2.2$ & $0.77(0.47-0.91)$ & 1.4 & 1.0 & 2.8 \\
\hline & 7 th & $-0.6 \pm 2.3$ & $-1.3 \pm 2.1$ & $0.75(0.40-0.90)$ & 1.4 & 1.0 & 2.8 \\
\hline & 8th & $-0.3 \pm 2.3$ & $-0.8 \pm 2.2$ & $0.29(-0.23-0.68)$ & 2.7 & 1.9 & 5.0 \\
\hline \multirow[t]{8}{*}{ Left diagonal } & $1 \mathrm{st}$ & $-0.0 \pm 3.7$ & $-0.6 \pm 3.2$ & $0.61(0.18-0.84)$ & 3.1 & 2.2 & 6.0 \\
\hline & 2 nd & $-1.0 \pm 1.8$ & $-1.1 \pm 2.2$ & $0.44(-0.07-0.77)$ & 2.2 & 1.5 & 4.3 \\
\hline & $3 \mathrm{rd}$ & $0.2 \pm 2.9$ & $-0.7 \pm 2.3$ & $0.60(0.18-0.84)$ & 2.3 & 1.6 & 4.5 \\
\hline & 4th & $0.7 \pm 2.3$ & $-0.6 \pm 2.3$ & $0.28(-0.16-0.66)$ & 2.7 & 1.9 & 5.3 \\
\hline & 5 th & $0.6 \pm 2.6$ & $0.4 \pm 2.8$ & $0.57(0.11-0.83)$ & 2.6 & 1.8 & 5.1 \\
\hline & 6th & $0.4 \pm 2.1$ & $-0.0 \pm 2.7$ & $0.23(-0.30-0.65)$ & 3.0 & 2.1 & 5.9 \\
\hline & 7 th & $-0.5 \pm 2.4$ & $-0.1 \pm 2.2$ & $0.38(-0.13-0.73)$ & 2.6 & 1.8 & 5.0 \\
\hline & 8th & $-0.2 \pm 2.3$ & $-1.0 \pm 2.3$ & $0.06(-0.42-0.53)$ & 2.9 & 2.1 & 5.7 \\
\hline
\end{tabular}

ICC intraclass correlation coefficient, 95\% CI 95\% confidence interval, SDd standard deviation of the difference between two measurement scores, SEM standard error of measurement, $M D C_{95}$ minimal detectable change with $95 \% \mathrm{CI}$
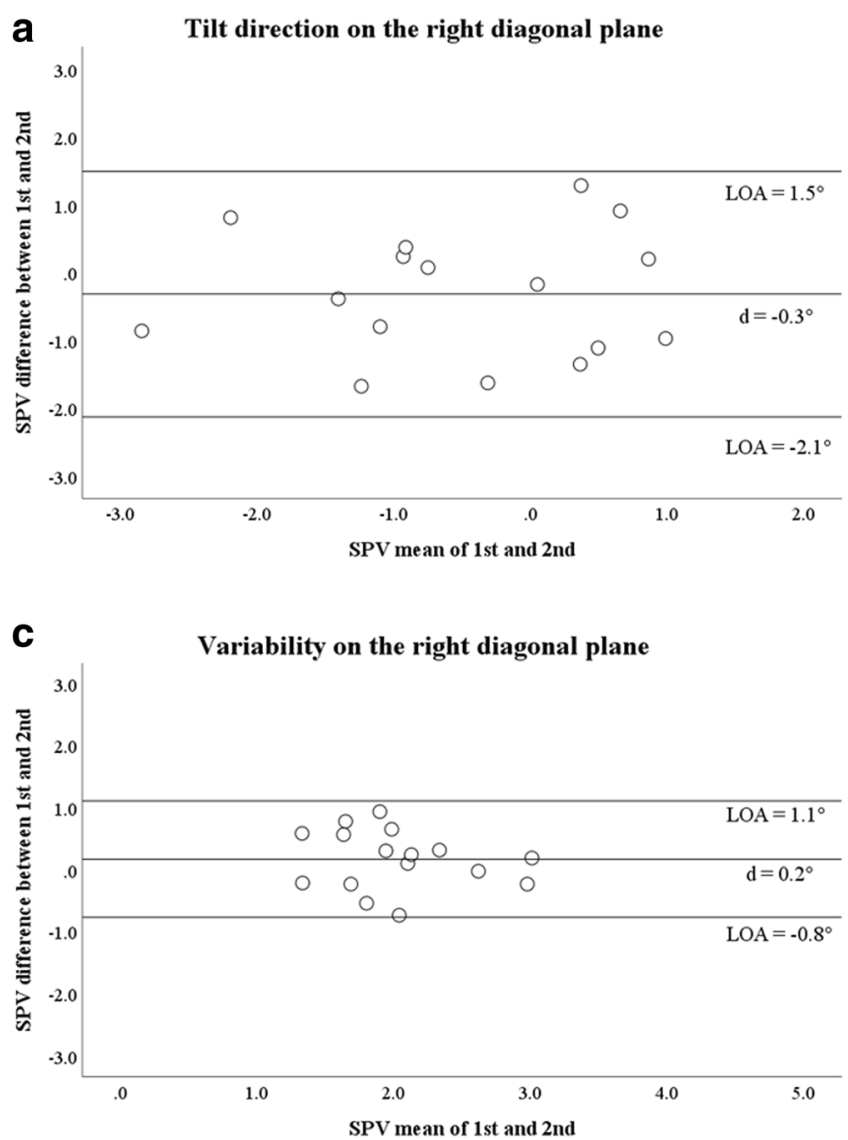

Fig. 3 Bland-Altman plots of the subjective postural vertical (SPV) on the right and left diagonal planes showing the differences between the first and second assessments and means of the first and second
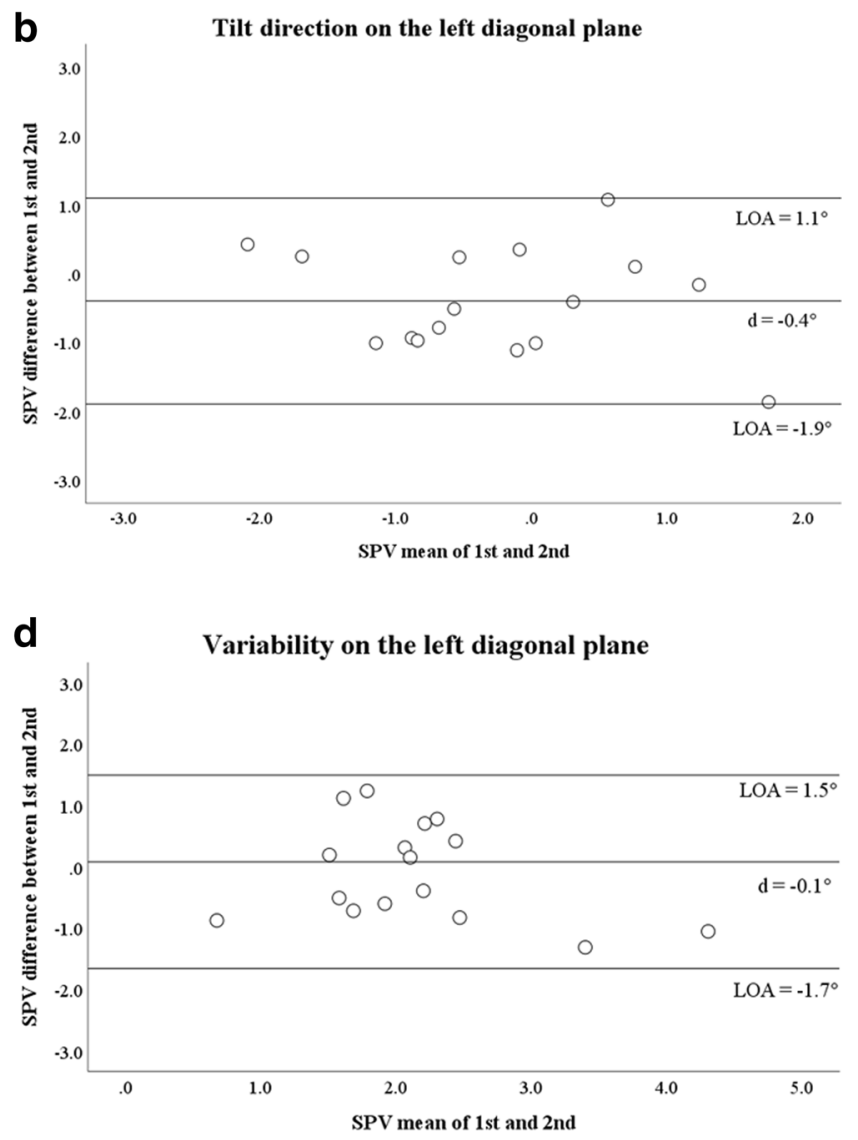

assessments, with the mean difference (d) and 95\% limits of agreement (LOA). SPV subjective postural vertical 
Table 4 Demographics of the young and elderly groups

\begin{tabular}{llll}
\hline Variable & Total $(\mathrm{n}=38)$ & Young $(\mathrm{n}=20)$ & Elderly $(\mathrm{n}=18)$ \\
\hline Age (years) & $37.9 \pm 19.7$ & $31.8 \pm 8.4$ & $67.0 \pm 9.4$ \\
Age (range, years) & $22-79$ & $22-49$ & $53-79$ \\
Gender (male/female) & $13 / 25$ & $8 / 12$ & $5 / 13$ \\
Height $(\mathrm{cm})$ & $162.0 \pm 9.4$ & $163.7 \pm 8.4$ & $160.1 \pm 10.5$ \\
Body weight $(\mathrm{kg})$ & $55.9 \pm 11.0$ & $55.6 \pm 10.6$ & $55.9 \pm 11.8$ \\
BMI $\left(\mathrm{kg} / \mathrm{m}^{2}\right)$ & $21.2 \pm 2.8$ & $20.6 \pm 2.1$ & $21.7 \pm 3.3$ \\
\hline
\end{tabular}

*Values are presented as mean \pm standard deviation.

$B M I$ body mass index

\section{Statistical analysis}

Test-retest reliability was calculated using the intraclass correlation coefficient (ICC) with $95 \%$ confidence interval $(95 \% \mathrm{CI})$ from a two-way mixed-effects model, with the mean of eight measurements and absolute agreement (Koo \& Li, 2016). Briefly, the measurement error was calculated using the minimal detectable change with $95 \% \mathrm{CI}\left(\mathrm{MDC}_{95}=\right.$ standard error of measurement $(\mathrm{SEM}) \times 1.96 \times \sqrt{2}$ ), which is determined from the standard deviation of the difference (SDd): $\mathrm{SEM}=\mathrm{SDd} \times \sqrt{ } 2$. To estimate SEM, SDd between the first and second assessments was calculated.

The agreement between the first and second assessments was determined by calculating the mean difference
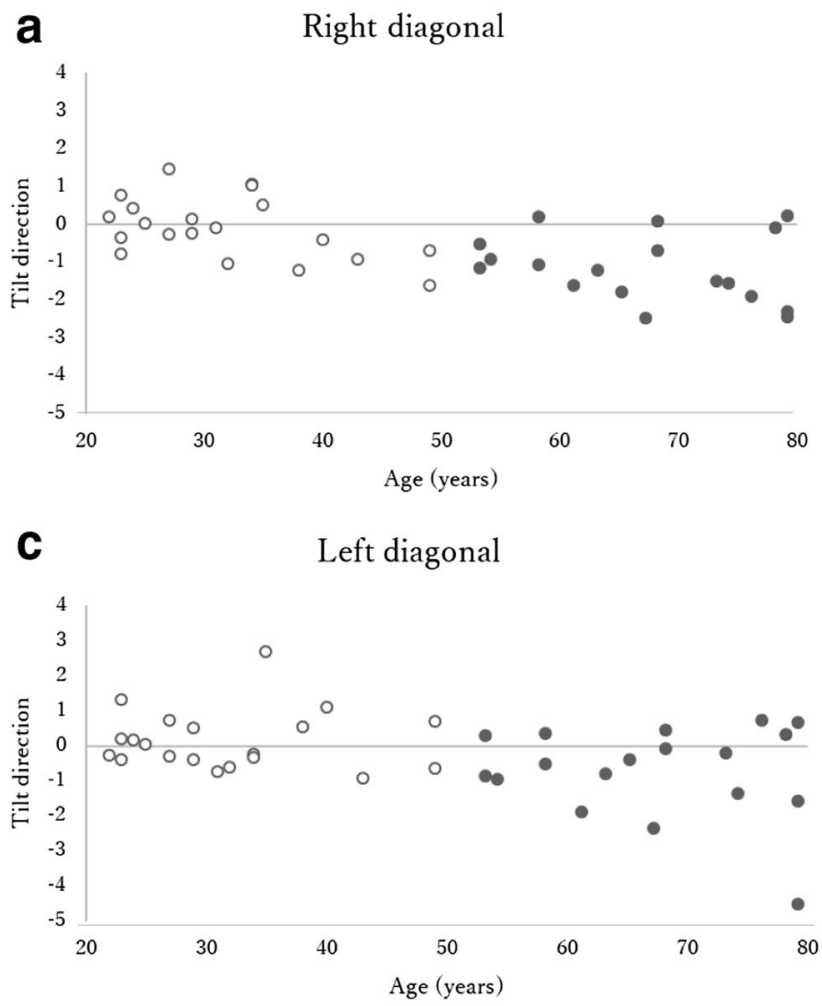

Fig. 4 Distribution of data for the subjective postural vertical (SPV). The tilt direction $(\mathbf{A}, \mathbf{C})$ and variability $(\mathbf{B}, \mathbf{D})$ in the SPV on the right or left between assessments (d) and 95\% limits of agreement (LOAs: $d \pm 1.96$ standard deviation [SD]), displayed in Bland-Altman plots (Bland \& Altman, 1986).

With respect to age-related change, demographic data were compared between the young and elderly groups using the unpaired t-test and chi-squared test. Statistical differences in SPV between the young and elderly groups were tested using the dependent two-group MannWhitney U test, because most parameters of tilt direction and variability were not normally distributed. Descriptive data are reported as mean with SD or, otherwise, as median with range. The Shapiro-Wilk test was used to determine data normality.

Statistical analysis was performed using PASW Statistics ver. 18.0 (SPSS Inc., Tokyo, Japan), with the level of significance set at $5 \%$.

\section{Results}

\section{Test-retest reliability}

Table 1 presents the demographic data of 16 healthy subjects, and Tables 2 and 3 show the results of the first and second assessments, as well as the ICC (95\% CI), SDd, $\mathrm{SEM}$, and $\mathrm{MDC}_{95}$. With respect to tilt direction, $\mathrm{ICC}_{1,1}$ and $\mathrm{MDC}_{95}$ were 0.69 (95\% CI: $0.33-0.88$ ) and $1.8^{\circ}$,
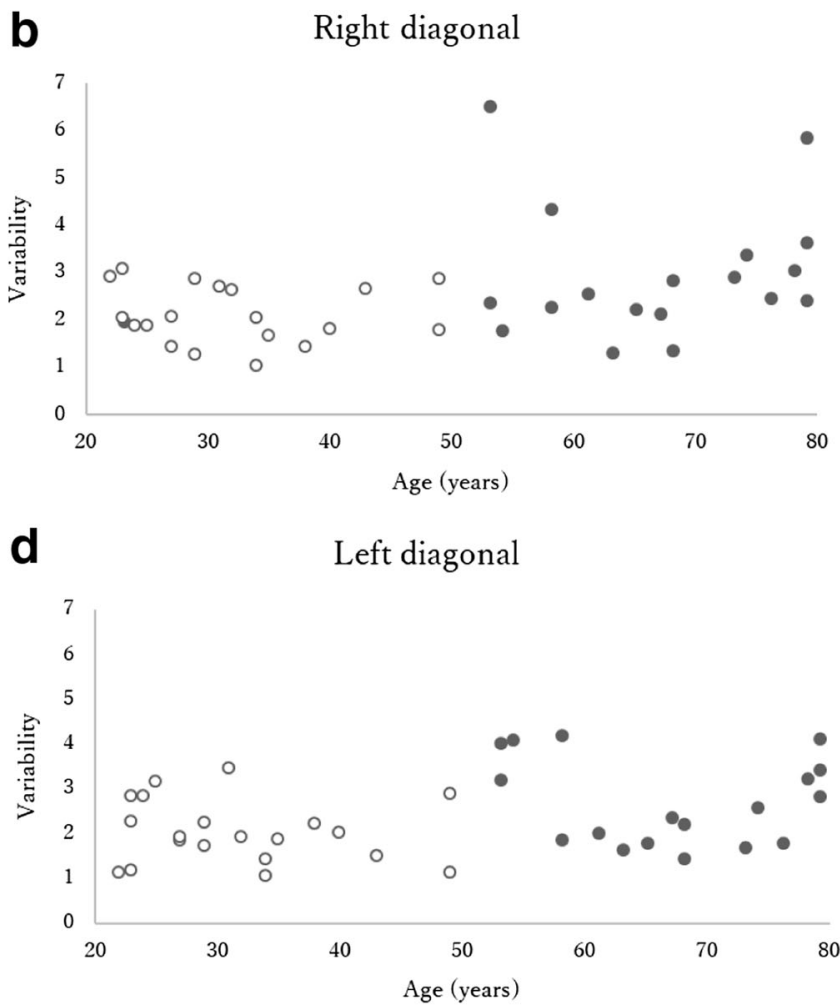

diagonal plane. The white dots represent healthy subjects aged $<50$ years, whereas the black dots represent healthy subjects aged $>50$ years 
Table 5 Age-related changes in subjective postural vertical on the diagonal plane

\begin{tabular}{llrrr}
\hline Variable & Condition & \multicolumn{1}{l}{ Total } & Young & Elderly \\
\hline Tilt direction $\left({ }^{\circ}\right)$ & Right diagonal & $-0.5(-1.3-0.2)$ & $-0.2(-0.8-0.5)$ & $-1.1(-1.7$ to -0.3$)$ \\
& Left diagonal & $-0.3(-0.7-0.5)$ & $-0.1(-0.4-0.6)$ & $-0.4(-1.4-0.4)$ \\
Variability $\left({ }^{\circ}\right)$ & Right diagonal & $2.4(1.8-2.9)$ & $2.0(1.7-2.7)$ & $2.6(2.2-3.5)$ \\
& Left diagonal & $2.1(1.7-3.0)$ & $1.9(1.4-2.7)$ & 0.002 \\
& & & & $2.5(1.8-3.6)$ \\
\hline
\end{tabular}

Values are presented as median (range); p-values indicate the results for the difference between the young and elderly groups.

respectively, on the right diagonal plane and 0.71 (95\% CI: $0.36-0.88$ ) and $1.5^{\circ}$, respectively, on the left diagonal plane. With respect to variability, $\mathrm{ICC}_{1,1}$ and $\mathrm{MDC}_{95}$ were 0.61 (95\% CI: $0.19-0.84)$ and $0.9^{\circ}$, respectively, on the right diagonal plane and 0.63 (95\% CI: 0.22-0.85) and $1.6^{\circ}$, respectively, on the left diagonal plane.

Bland-Altman plots indicated no systematic bias as the degree concerning mean difference was distributed (Fig. 3).

\section{Age-related changes}

The characteristics of the young and elderly groups are described in Table 4. There were no differences in gender (chi-squared [1] $=0.629, \mathrm{p}=0.428$ ), height ( $\mathrm{t}[36]=1.167$, $\mathrm{p}=0.251$ ), body weight ( $\mathrm{t}[36]=-0.101, \mathrm{p}=0.920)$, and BMI ( $\mathrm{t}[36]=-1.305, \mathrm{p}=0.200$ ) between the groups. The distribution of tilt direction and variability in all subjects are shown in Fig. 4. Age-related changes are presented in Table 5. On the right diagonal plane, the elderly group exhibited a tendency for backward tilt toward the left side $(p=0.083)$ and high variability compared with the young group $(\mathrm{p}<0.05)$. On the left diagonal plane, the elderly group showed a significant backward deviation toward the right side compared with the young group $(\mathrm{p}<0.05)$. We found that the elderly group exhibited a significantly higher variability in measurement $(\mathrm{p}<0.05)($ Table 5$)$.

\section{Differences on the right and left diagonal planes}

With respect to the comparison on the right and left diagonal planes, no significant difference in tilt direction $(\mathrm{p}=0.322)$ and variability $(\mathrm{p}=0.765$ ) on the right and left diagonal planes was observed in the young group, whereas in the elderly group, the tilt direction indicated a significant diagonally backward deviation on the right diagonal plane compared with that on the left diagonal plane $(p<0.038)$, and there was no significant difference in variability $(\mathrm{p}=0.286)$.

\section{Discussion}

To our knowledge, this is the first study to assess SPV on the diagonal plane in healthy subjects. The test-retest reliability was 0.61 or more when measured on the right and left diagonal planes. Bergmann et al. (2015) analyzed the reliability of SPV in subjects standing on the frontal and sagittal planes and reported that the ICCs ranged from 0.6 to 0.7 . Although the measuring position was different to that in previous studies, the SPV measurement using the EVB in the present study showed the same degree of reliability. Moreover, our results indicated a large 95\% CI of ICC values that ranged from $0.19-0.36$ to $0.84-0.85$, suggesting poor to good reliability according to Koo et al. (2016). Based on the 95\% CI, Koo et al. (2016) described that ICC values less than 0.5 , between 0.5 and 0.75 , between 0.75 and 0.9 , and greater than 0.9 are indicative of poor, moderate, good, and excellent reliability, respectively. Moreover, in all trials, there exists the possibility that the variation in reliability values and the large SEM also increases the $95 \%$ CI. Moreover, one of the reasons for the large 95\% CIs of all ICCs is the subjective aspect of assessment; thus, subject factors such as mental fatigue or ability to concentrate may also have a major impact. In the future, it may be necessary to adjust the number of measurements and take a break, taking the subject's mental load into consideration. However, these values were comparable to the $95 \%$ CIs of ICC values reported by Bergmann et al. (2015), which ranged from 0.23-0.40 to 0.85-0.88; hence, a large $95 \%$ CI may be one of the features of research on SPV.

The $\mathrm{MDC}_{95}$ is an index showing an error of change when it is repeatedly measured. If the obtained value is equal to or less than the $\mathrm{MDC}_{95}$, then it is within the error range (de Vet et al., 2006). However, if the value is greater than the $\mathrm{MDC}_{95}$, it can be determined that a true change has occurred (de Vet et al., 2006). In this study, the $\mathrm{MDC}_{95}$ was observed to be $0.9^{\circ}$ to $1.8^{\circ}$, which is a useful index for judging the time course change and therapeutic effect in the study of SPV.

In the elderly group, the tilt direction of SPV shifted diagonally backward; further, the variability was higher in the elderly group than in the young group on both the right and left diagonal planes. Barbieri et al. (2010) investigated the SPV on the sagittal plane using a unique measuring device called the wheel paradigm in healthy subjects, and reported that the SPV showed a significantly greater backward tilt in 
elderly subjects than in young subjects and a greater SPV range with increasing age. Bisdorff et al. (1996) observed a larger sector of SPV with aging on the roll and pitch plane in healthy subjects and reported a decline in the sensitivity of perception of body verticality. Fukata et al. (2017) assessed the SPV on the frontal plane and reported no difference in the tilt direction between the elderly and young subjects; however, the variability was significantly higher in elderly subjects than in young subjects. Therefore, the diagonal backward deviation on the diagonal plane may include elements of the tilt direction on the sagittal plane. Moreover, the high variability on the diagonal plane may reflect the decline in somatosensory and vestibular inputs with increasing age (Bruce, 1980; Rosenhall, 1975). In contrast, no significant difference in the tilt direction on the left diagonal plane was noted between the young and elderly groups. In addition, the elderly group showed a significant diagonally backward deviation on the right diagonal plane compared with that on the left diagonal plane. The internal model is modulated or updated by the mutual influence of the sensory and motor systems (Barra, 2012). As the subjects in the present study were all right-handed, there exists the possibility that the postural orientation ability of the left space, which is a subordinate hemisphere, is relatively lower than that of the right space. That is, the spatial cognitive ability in the left backward direction possibly declines, which might be remarkable in the elderly group. These findings may be important information in considering the effect of handedness in subjective vertical research. However, as nonparametric testing is used for data analysis, the low detection power of data is considered a factor that is not different. In the future, it may be necessary to further increase the number of subjects being examined.

This study has some potential limitations concerning SPV measurement on the diagonal plane using the EVB. First, the machine provides a great deal of tactile information. Says et al. (2012) investigated the relationship between somatosensory loss and perception of verticality in stroke patients. They found that the absolute value of SPV was significantly related to both joint-related and skinrelated somatosensory information. When the EVB is tilted diagonally backward, the body is in contact with the back and side sheets. In this manner, the body's vertical axis may posteriorly and diagonally deviate in response to extensive somatosensory information. Moreover, when the EVB is tilted anteriorly and diagonally, only unilateral sensory information is obtained. Therefore, tactile cues might considerably affect the direction and sensitivity of SPV. Thus, we believe that it is also necessary to consider the characteristics of the measuring equipment itself. Second, the inter-rater reliability between experimenters has not yet been determined for the EVB, and using only one experimenter for SPV measurement may have limited the generalizability of this study. Finally, the functional value of
SPV on the diagonal plane is unclear. Further studies investigating whether the SPV on the diagonal plane affects balance in elderly subjects and patients with cerebrovascular disease are required. Moreover, to clarify the functional value of SPV on the diagonal plane, it is important to investigate the relationship between SPV on the diagonal plane and that on the frontal and sagittal planes.

In conclusion, the reliability of SPV measurement on the diagonal plane using the EVB has been shown to be good. In addition, we determined the measurement error, which may be a useful index for the SPV because it changes over time in response to treatment. Furthermore, as we showed changes due to aging, future studies on SPV on the diagonal plane in patients with neurological disorders, including cerebrovascular disease, must take changes due to aging into account.

Funding This research was supported by funding from the Hidaka Project (28-D-1-09) at Saitama Medical University International Medical Center.

\section{Compliance with ethical standards}

Conflict of interest The authors declare no conflicts of interest.

Publisher's Note Springer Nature remains neutral with regard to jurisdictional claims in published maps and institutional affiliations.

\section{References}

Baggio, J. A., Mazin, S. S., Alessio-Alves, F. F., Barros, C. G., Carneiro, A. A., Leite, J. P., ..., Santos-Pontelli, T. E. (2016). Verticality perceptions associate with postural control and functionality in stroke patients. PLOS ONE, 11(3), e0150754.

Barbieri, G., Gissot, A. S., \& Pérennou, D. (2010). Ageing of the postural vertical. Age, 32(1), 51-60.

Barra, J., Pérennou, D., Thilo, KV., Gresty, MA., Bronstein, AM. (2012). The awareness of body orientation modulates the perception of visual vertical. Neuropsychologia, 50(10), 2492-2498.

Bergmann, J., Kreuzpointner, M. A., Krewer, C., Bardins, S., Schepermann, A., Koenig, E., ...Jahn, K. (2015). The subjective postural vertical in standing: Reliability and normative data for healthy subjects. Attention, Perception, \& Psychophysics, 77(3), 953-960.

Bisdorff, A. R., Wolsley, C. J., Anastasopoulos, D., Bronstein, A. M., \& Gresty, M. A. (1996). The perception of body verticality (subjective postural vertical) in peripheral and central vestibular disorders. Brain, 119(5), 1523-1534.

Bland, J. M., \& Altman, D. G. (1986). Statistical methods for assessing agreement between twomethods of clinical measurement. Lancet, 1 , 307-310.

Bruce, M. F. (1980). The relation of tactile thresholds to histology in the fingers elderly. Journal of Neurology, Neurosurgery and Psychiatry, 43(8), 730-734.

de Vet, H. C., Terwee, C. B., Knol, D. L., \& Bouter, L. M. (2006). When to use agreement versus reliability measures. Journal of Clinical Epidemiology, 59(10), 1033-1039.

Fukata, K., Amimoto, K., Fujino, Y., Inoue, M., Inoue, M., Takahashi, Y., \& Takahashi, H. (2017). The effects of aging on the subjective 
vertical in the frontal plane in healthy adults. Journal of Physical Therapy Science, 29(11), 1950-1953.

Karnath, H. O., Ferber, S., \& Dichgans, J. (2000). The origin of contraversive pushing: Evidence for a second graviceptive system in humans. Neurology, 55(9), 1298-1304.

Karnath, H. O., \& Broetz D. (2003). Understanding and treating "pusher syndrome". Physical Therapy, 83(12), 1119-1125.

Koo, TK., Li MY. (2016). A guideline of selecting and reporting intraclass correlation coefficients for reliability research. J Chiropr Med, 15(2), 155-163.
Pérennou, D. A., Mazibrada, G., Chauvineau, V., Greenwood, R., Rothwell, J., Gresty, M. A., \& Bronstein, A. M. (2008). Lateropulsion, pushing and verticality perception in hemisphere stroke: A causal relationship? Brain, 131(9), 2401-2413.

Rosenhall, U., \& Rubin, W. (1975). Degenerative changes in the human vestibular sensory epithelia. Acta Otolaryngology, 79(1-2), 67-80.

Saeys, W., Vereeck, L., Truijen, S., Lafosse, C., Wuyts, F. P., \& Van de Heyning, P. (2012). Influence of sensory loss on the perception of verticality in stroke patients. Disability and Rehabilitation, 34(23), 1965-1970. 